\title{
An evaluation of the use of a novel microbicidal liquid polymer for the reduction of pin-tract infection in external fixation procedures for deformity correction and traumatic provisional fixation
}

\author{
Shital Pema* \\ Kettering Health System, Ohio, USA
}

\begin{abstract}
Objective: To evaluate whether the use of a microbicidal liquid polymer, forming an occlusive elastomeric barrier when applied as part of a standard pin-tract care protocol leads to a reduction in pin-tract infections.

Methods: This IRB approved randomized prospective study included 12 patients who received external fixation following deformity correction. Patients received 10 pins each for a total of 120 pins (60 pins per group). Six patients received standard pin-tract care, while 6 patients received identical pin-tract care with the addition of the microbicidal liquid polymer applied at the conclusion of each care regimen. Data was collected through examinations by clinical staff under supervision of the principal investigator. Data was categorical and analyzed using chi-square or Fisher exact tests to measure variable independence. Results were analyzed on a per patient and per pin basis.

Results: Results showed a statistically significant reduction in pin-tract infections for pins of the microbicidal liquid polymer group (14 infected pins in standard care group vs 0 infected pins in microbicidal liquid polymer group, $\mathrm{p}<0.001)$. For the patient-level portion, two patients (33.3\%) in the standard care group experienced infection while none $(0 \%)$ in the microbicidal liquid polymer group experienced infection.

Conclusions: The application of a microbicidal liquid polymer led to a statistically significant reduction in pin-tract infections. The use of this 510 ( $\mathrm{k}$ ) cleared product as an addition to pin-tract care could have a dramatic impact on the increased adoption of external fixation.
\end{abstract}

\section{Introduction}

Metal pins and wires are commonly used for external fixation in patients undergoing orthopaedic reconstructive surgery for deformity correction or trauma. These pins or wires protrude through the skin and create an avenue for migration of organisms. The disruption of the dermal barrier increases the risk of infection. Pin-site infections are a very common complication of external fixation, and unfortunately, the literature is scant on how to optimally minimize the risk of infection.

\section{Background}

A Cochrane Collaboration Review published in 2004 to assess the effect on infection rates of different methods of cleaning and dressing orthopedic percutaneous pin sites determined that there was little evidence as to which pin-site care regimen best reduce infection rates [1].

Depending on the reference, pin-tract infections rates have been reported to range from $0 \%$ to $100 \%$. A systematic review of 150 Journal Articles covering 6130 total patients from 1980 through 2014 showed the incidence of pin-tract infections associated with external fixation published in 2016 revealed a cumulative pin-tract infection rate of $27 \%$. This rate was defined as the inherent risk of any given research participant developing a pin-tract infection at a random pin or wire site during the course of treatment with external fixation [2]. Another 2016 review described pin-tract infection as the most common expected problem, or even an almost inevitable complication when external fixation was used [3].

Several limitations of the current literature pose significant barriers to the study of pin-site infection preventative strategies. Limitations include lack of uniform definition/criteria to diagnose and classify severity of pin-tract infections, which makes it difficult to define the true incidence. Highly variable control groups between studies as well as non-uniform risk factors between groups in the same study, which creates difficulty in applying study results to an individual practice or protocol for preventative care. Within studies, treatment groups often differ by more than one variable (e.g. changing both the cleaning solution and dressing types), which makes it impossible to determine the effect of individual variables and applying study results to an individual practice for protocol. There exist few randomized controlled trials, which creates clinical practice based on low quality, underpowered and potentially biased studies. There is no consistency

${ }^{\star}$ Correspondence to: Shital Pema, Kettering Health System, Ohio, USA, E-mail: shital.pema108@gmail.com

Key words: infection prevention, microbicidal, pin-tract infection, MRSA prevention, non-antibiotic, wound care, killing pathogens, wound healing

Received: September 10, 2019; Accepted: September 28, 2020; Published: October 05,2020 
Pema S (2020) An evaluation of the use of a novel microbicidal liquid polymer for the reduction of pin-tract infection in research participants receiving external fixation following deformity correction and traumatic provisional fixation

in reporting-infection rates (per patient vs. per individual pin site), which creates difficulty in studying incidence, comparing studies or conducting an accurate meta-analysis [4].

Although a variety of organisms may be involved in pin-tract infections, Staphylococcus Aureus is the most common pathogen isolated from implant-associated osteomyelitis and over $50 \%$ of cases are caused by hard-to-treat methicillin-resistant S. aureus (MRSA) strains [5]. Biofilm-related bacterial infections have been recognized as being exceedingly difficult to treat with conventional systemic antibiotic therapies. Inhibiting bacterial adhesion appears to be a promising strategy in avoiding biofilm formation [6]. The utilization of a microbicidal liquid that eradicates all organisms on contact, regardless of antibiotic sensitivity, and forms a protective barrier should provide benefit in reducing infections at pin-tract sites.

\section{Methods}

Twelve research participants eligible for the study were those undergoing deformity correction and traumatic provisional fixation. These research participants each had 10 pins placed to ensure the required rigid fixation. The number of external fixation pins totalled 120 pins.

This principle investigator had previously utilized the microbicidal liquid polymer (Preventogen, Prevent-Plus, St. Louis, MO) which eradicates all organisms it comes in contact with, creating a protective film barrier over clean dry wounds as an additional step in the treatment protocol for several reconstructive surgery patients (approximately 25) with over 200 pin-sites with only 2 pin-site infections. This represented a significant reduction from the average incidence of pin site infections reported in the literature (approximately $8 \% v s .27 \%$ on a per patient basis). A recently published retrospective case series evaluating this microbicidal liquid polymer dressing reported on 6 patients and 66 pins with no infections when the microbicidal liquid polymer was used as an addition to a standard pin-site care regimen [7].

A total of 12 research participants were selected. Research participants with 60 pin-sites (10 pins/research participant) were enrolled into the study for the control group receiving standard pintract care. Six research participants with 60 pin-sites (10 pins/research participant) were enrolled into the study for the microbicidal liquid polymer group. The microbicidal liquid polymer group received the same standard pin-tract care with the addition of the microbicidal liquid polymer applied at each cleaning of the pin-tract area as an additional step in the pin-tract care protocol.

The age of research participants was chosen based on the majority of the principal investigator's research participants being adults and to avoid any special consideration that may apply to research with minors. The study was not gender specific. There were no enrollment restrictions based upon race or ethnic origin. Inclusion criteria included deformity correction, traumatic provisional fixation, 18 years of age or greater and no known contraindication to receive the microbicidal liquid polymer. Exclusion criteria included age less than 18 years, known allergy to methylene chloride, known sensitivity to organic polymers, non-clean dry wound at pin and vulnerable research participant (institutionalized, students, employees, prisoners, or those with decisional incapacity, etc.).

Thirteen patients were enrolled into the study. One patient in the microbicidal liquid polymer group dropped out due to severe venous stasis which caused her pins to loosen, necessitating removal of all pins. The principle investigator determined it was unrelated to the microbicidal liquid polymer or any part of the study protocol, so the patient was removed from the study. Twelve total research participants who were randomized using a computer-generated randomization tool to either the control group or the microbicidal liquid polymer group (6 participants per group) with 120 pin-sites (60 pins per group) completed the study. Each potential research participant undergoing deformity correction and/or traumatic provisional surgery were asked to participate in the study to evaluate if the topical application of the microbicidal liquid polymer as an additional step in the management of post-operative pin-tract site care would reduce the incidence of pintract infections (Table 1).

For the control group, the usual care of pin-tract sites was followed as outlined below (Pin-tract Care Regimen). For the microbicidal liquid polymer group, the same care regimen was followed with the addition of the microbicidal liquid polymer being applied topically to pin-tract sites as the experimental procedure being performed for research purposes. No additional tests or procedures outside of the existing standard regimen were utilized.

\section{Product description}

Preventogen is an FDA cleared Class One medical device. This organic polymer, when applied to the disrupted skin in liquid form, eradicates all organisms on contact, regardless of antibiotic sensitivity. It then quickly transforms into an elastomeric, transparent, biodegradable and occlusive dressing. The product has been studied and used on hundreds of wounds secondary to trauma, shave biopsies, and Mohs micrographic surgery, including on flap and full-thickness skin graft repairs.

\section{In vitro testing}

Laboratory tests were conducted to determine the microbicidal activity of for FDA clearances. Additional in vitro testing was performed to evaluate the antimicrobial properties of Preventogen using In vitro Time Kill Method vs. the following Bacterial: Escherichia coli, Pseudomonas aeruginosa, Staphylococcus aureous MRSA, VRE, Acinetobacter baumannii, Bacillus subtilis, Clostridium sporogenes, Streptococcus pyogenes; Fungal: Candida auris, Aspergillus niger, T. rubrum, Candida albicans, Aspergillus brasiliensis; Viral: Herpes Simplex 1, Herpes Zoster; All testing was done in accordance with Good Laboratory Practices as specified in 21 CFR Part 58. All tests provided satisfactory results indicating that the Elastic Skin Liquid Polymer is safe and effective. In all tests, the bacteria, fungi and viruses were killed $100 \%$ within 3 minutes (Table 2).

\section{Pin-tract care regimen}

All patients' pins and wire sites were cleaned daily while hospitalized. Basic pin care was performed once daily by hospital nursing staff prior to discharge. Following discharge from the hospital, the pin-tract care protocol was continued by the research participant's caregiver.

Pin-sites were cleaned when the surrounding skin and gauze were soft. This made the removal of gauze and cleaning of pins less painful. The purpose of the cleaning was to prevent the skin from attaching to the pins and wires, and to decrease the chance of infection. The area was then inspected for any signs of infection.

\section{Control group procedure}

Step 1: Brush the pin-sites with saline using an ordinary soft toothbrush or gauze with sterile gloves 
Pema S (2020) An evaluation of the use of a novel microbicidal liquid polymer for the reduction of pin-tract infection in research participants receiving external fixation following deformity correction and traumatic provisional fixation

Table 1. Characteristics of Patients enrolled for the study

\begin{tabular}{|c|c|c|c|c|}
\hline Group & Age & Gender & Comorbidities & Infection (Y/N) \\
\hline Control & 67 & M & $\begin{array}{l}\text { CAD, LVD, HCL, arrythmia, Type II DM, HTN, hx of PE, diabetic } \\
\text { ulcer rt foot }\end{array}$ & $\mathrm{N}$ \\
\hline Control & 51 & F & HTN, edema, GERD, HLD, previous history of surgery, obesity & $\mathrm{N}$ \\
\hline Control & 41 & M & $\begin{array}{l}\text { foot ulcer, exercise intolerance, methamphetamine abuse, OM of rt } \\
\text { foot and ankle, PN }\end{array}$ & $\mathrm{N}$ \\
\hline Control & 50 & F & $\begin{array}{l}\text { IDDM, HTN, HLD, GERD, morbid obesity, edema, OA, thyroid } \\
\text { disease, previous history of surgery }\end{array}$ & $\mathrm{Y}$ \\
\hline Control & 64 & M & $\begin{array}{l}\text { HTN, hypothyroidism, smoker, HLD, morbid obesity, edema, } \\
\text { previous history of surgery }\end{array}$ & Y \\
\hline Control & 38 & F & $\begin{array}{l}\text { IDDM with h/o DKA, gastroparesis, Rt foot ulcer, diabetic } \\
\text { retinopathy, HLD, h/o OM left toe }\end{array}$ & $\mathrm{N}$ \\
\hline Microbicidal Liquid Barrier & 62 & F & $\begin{array}{l}\text { HTN, cardiac arrythmia, Type II DM (uncontrolled), HLD, PVD, } \\
\text { morbid obesity, PN }\end{array}$ & $\begin{array}{l}\text { N: Patient experienced } \\
\text { loosening of pins due to edema. } \\
\text { Pins removed with no infection. } \\
\text { Unable to complete study. }\end{array}$ \\
\hline Microbicidal Liquid Barrier & 49 & $\mathrm{~F}$ & IDDM, HTN, hypothyroid, former smoker & $\mathrm{N}$ \\
\hline Microbicidal Liquid Barrier & 64 & $\mathrm{~F}$ & $\begin{array}{l}\text { Type II DM, Diabetic ulcer of right mid foot, history of non-healing } \\
\text { wound, Sjogren's syndrome, PVD, HTN, HLD }\end{array}$ & $\mathrm{N}$ \\
\hline Microbicidal Liquid Barrier & 45 & M & $\begin{array}{l}\text { CKD, ESRD, IDDM with h/o DKA, HTN, HLD, PVD, RA, } \\
\text { osteomyelitis, s/p amputation less toe - right } 4 \text { th, PN }\end{array}$ & $\mathrm{N}$ \\
\hline Microbicidal Liquid Barrier & 60 & M & HTN, IDDM, diabetic retinopathy, IHD & $\mathrm{N}$ \\
\hline Microbicidal Liquid Barrier & 32 & M & $\begin{array}{l}\text { HTN, morbid obesity, left lower extremity cellutis, Type II DM, } \\
\text { history of MRSA rt foot, HLD, asthma }\end{array}$ & $\mathrm{N}$ \\
\hline Microbicidal Liquid Barrier & 74 & M & HTN, history of prostate CA, HLD, RA & $\mathrm{N}$ \\
\hline \multicolumn{5}{|c|}{$\begin{array}{l}\text { CAD: Coronary Artery Disease; LVD: Left Ventricular Dysfunction; HCL: Hypercholesterolemia; DM: Diabetes Mellitis; HTN: Hypertension; PE: Pulmonary Embolism; GERD: } \\
\text { Gastroesophageal Reflux Disease; HLD: Hyperlipidemia; OM: Osteomyelitis; PN: Peripheral Neuropathy; IDDM: Insulin Dependent Diabetes Mellitis; OA: Osteoarthritis; DKA: } \\
\text { Diabetic Ketoacidosis; PVD: Peripheral Vascular Disease; CKD: Chronic Kidney Disease; ESRD: End Stage Renal Disease, IHD: Ischemic Heart Disease; MRSA: Methicillin Resistant } \\
\text { Staphyloccus aureus, } \\
\text { CA: Cancer; RA: Rheumatoid Arthritis }\end{array}$} \\
\hline
\end{tabular}

Table 2. In vitro tests conducted to determine the microbicidal activity for FDA clearances

\begin{tabular}{|c|c|c|c|c|}
\hline Organism & Common & Log Reduction & Day $1 \%$ Reduction & Day $2 \%$ Reduction \\
\hline Candida albicans & Fungus & 1 & $>99.9999 \%$ & $>99.9999 \%$ \\
\hline Staphylococcus aureus (MRSA) & Bacteria & 5.2 & $>99.9999 \%$ & $>99.9999 \%$ \\
\hline Pseudomonas aeruginosa & Bacteria & 5.3 & $>99.9999 \%$ & $>99.9999 \%$ \\
\hline \multicolumn{5}{|c|}{ Additional In vitro results post $510(\mathrm{k})$ clearance } \\
\hline Organism & Common & Log10 Reduction & 5 Minutes \% Reduction & \\
\hline Enterococcus faecalis & Bacteria & $>4.5$ & $>99.99 \%$ & \\
\hline Staphylococcus aureus (MRSA) & Bacteria & 5.3593 & $>99.99 \%$ & \\
\hline Acinetobacter baumanni & Bacteria & 6.0065 & $>99.99 \%$ & \\
\hline Streptococcus pyogenes & Bacteria & 5.5924 & $>99.99 \%$ & \\
\hline Aspergillis brasiliensis & Mold & 8.375 & $99.99 \%$ & \\
\hline Candida albicans & Mold & 1.4425 & $99.99 \%$ & \\
\hline Escherichia coli & Bacteria & 1.6 & $99.99 \%$ & \\
\hline Pseudomonas aeruginosa & Bacteria & 1.28 & $99.99 \%$ & \\
\hline Herpes Simplex $1 \& 2$ (cold sores) & Virus & 5 & $>99.99 \%$ & \\
\hline Herpes Zoster (Shingles) & Virus & 4 & $>99.99 \%$ & \\
\hline
\end{tabular}

Step 2: If debris remains after step 1, use forceps (tweezers) to gently remove debris.

\section{Microbicidal liquid Polymer group additional procedure}

Step 3: Following steps 1 and 2, apply the microbicidal liquid polymer with a Q-tip on clean dry wound (extending approximately one inch around pin-site) and on the pin. To be applied at a minimum of at least three times a week and daily while in the hospital (Table 3 ).

The microbicidal liquid polymer is reapplied at the end of each pin care cleaning regimen.

\section{Statistical analysis}

This was a randomized prospective observational case series study to measure the potential reduction in pin-tract infection rate due to the utilization of a microbicidal liquid polymer which forms an elastomeric, occlusive barrier amongst research participants and individual pins for patients undergoing deformity correction and traumatic provisional fixation.

Weekly data was collected by clinical staff under supervision of the principal investigator. Data collected was primarily categorized and analyzed using tabulations (tables of frequencies), visual displays (histograms), and chi-square or Fisher exact tests to measure variable independence (e.g., infection rate based on age, number of pins/research participants, etc.).

All research participants were monitored for potential redness or irritation at the pin-tract sites, and if any were to occur, discontinuation of the application was considered if it was believed to be a result of a reaction to the product [8]. 
Pema S (2020) An evaluation of the use of a novel microbicidal liquid polymer for the reduction of pin-tract infection in research participants receiving external fixation following deformity correction and traumatic provisional fixation

Investigational review board (IRB) approval was obtained and informed research participant consent was obtained from each research participant.

\section{Results}

Results were analyzed on a per patient and per-pin basis. Patients undergoing deformity correction surgery had comparable risk factors across all patient groups. The groups were balanced for age, with slightly more females enrolled in the control group (50\% vs. 33\%). The results showed a statistically significant reduction in pin-tract infections for pins of the group receiving the microbicidal liquid polymer when compared to the pins in the group not receiving the microbicidal liquid polymer.

For the patient-level analysis, the response variable was whether the patient developed an infection (i.e. had at least one infected pin). The results are provided in Table 4. Two of 6 patients in the control group experienced infection (33.3\%) while none of 6 patients in the microbicidal liquid polymer group experienced infection ( $0 \%)$. Fisher's exact test was used to analyze the two variables (group and infection). Based on a $p$-value of 0.45 , there is not sufficient evidence to suggest a significant association between group and infection. Note that a prior power analysis indicated that a minimum sample size required to achieve $80 \%$ power was 33 patients per group. Due to lack of 66 available patients, this portion of the analysis is under-powered. However, from a descriptive standpoint, the results were in line with the values that went into the power analysis. If the observed trend continued with the appropriate sample size, it would become statistically significant.

For the pin-level analysis, the response variable is whether the individual pin-site became infected. The results are given in Table 5. Chi-square test was used to evaluate the two variables in this portion. The first variable being group (microbicidal liquid polymer or control), the second variable being infection at pin-site (60 pins in each group). Zero of 60 pins $(0 \%)$ were infected in the microbicidal liquid polymer group while 14 of 60 pins (23.3\%) were infected in the control group. Based on a $p$-value of $<0.0001$, there is strong evidence to suggest there is a significant association between group and infection.

Table 3. Study types

\begin{tabular}{|l|c|c|c|}
\hline & Initial Visit & $\begin{array}{c}\text { Day of } \\
\text { Hospital } \\
\text { Discharge }\end{array}$ & $\begin{array}{c}\text { Pin } \\
\text { Removal }\end{array}$ \\
\hline Informed consent obtained & $\checkmark$ & & \\
\hline Instruction on Pin-tract Care & & $\checkmark$ & \\
\hline Instruction on Signs and Symptoms of infection & & $\checkmark$ & \\
\hline $\begin{array}{l}\text { Final visit and final determination of infection } \\
\text { or no infection }\end{array}$ & & & $\checkmark$ \\
\hline
\end{tabular}

Table 4. Patient infection outcomes

\begin{tabular}{|l|c|c|c|}
\hline & \multicolumn{3}{|c|}{ Infection } \\
\hline Group & Yes & No & Percent Infected \\
\hline Microbicidal liquid polymer & 0 & 6 & 0 \\
\hline Control & 2 & 4 & 33 \\
\hline
\end{tabular}

Table 5. Pin infection outcomes

\begin{tabular}{|l|c|c|c|}
\hline & \multicolumn{3}{|c|}{ Infection } \\
\hline Group & Yes & No & Percent Infected \\
\hline Microbicidal liquid polymer & 0 & 60 & 0 \\
\hline Control & 14 & 46 & 23 \\
\hline
\end{tabular}

\section{Discussion}

\section{Limitations}

One limitation of this research includes the small sample size when considering per patient population which led to a non-statistically significant difference in the infection rate among the two patient groups.

\section{Implications for practice}

Financial costs of treating surgical site infections (SSIs) are on the rise. The number of surgical procedures performed continues to increase, and it is becoming more common for surgical patients to present with increasingly complex comorbidities [9]. It is estimated that approximately half of SSIs are deemed preventable using evidencebased strategies. The estimated average cost of a surgical site infection (SSI) can be more than $\$ 25,000$, increasing to more than $\$ 90,000$ if the SSI involves a prosthetic implant. Overall, SSIs cost the US healthcare system an estimated $\$ 3.5$ to $\$ 10$ billion annually [9]. There were 110,330 external fixation devices applied in the US in 2016 [10]. With an average infection rate of $27 \%$, that correlates to approximately 29,789 external fixation patients having a likelihood of pin-tract infection. Couple that with an average cost of $\$ 25,000$ for an SSI, the cost to the US healthcare system in 2016 was potentially as high as $\$ 744$ million.

Pin-tract infections continue to be a troublesome problem for patients of surgeons performing external fixation. Pin care must include collaboration among all health care professionals involved in the patient's care as well as the patients themselves. Optimally, pin care should begin before the patient enters the operating room. The overall health of the patient's soft tissue envelope should be assessed preoperatively [11]. Other risk factors for infection may include smoking, steroid treatment, perioperative blood transfusion, hypoalbuminemia (established nutritional marker), poor glucose control, or a history of leukopenia resulting from hematologic disease or iatrogenic bone marrow suppression.

Assessment should include a plan for anticipated pin and wire placement, the quality of the bone needs to be evaluated, the optimal size of pins or wires should be determined prior to surgery and preplanning the number and position of the pins/wires. All of these techniques will help to avoid an unstable frame which can lead to loosening, inflammation and eventually infection [11].

Once in the operating room, meticulous surgical technique is mandatory. An atraumatic insertion of the pin or wire through the skin, soft tissue envelope and bone is the goal. Some tips for reduction of trauma have been suggested. These include avoiding the generation of heat during the insertion process, inserting wires using a sponge soaked in alcohol to cool and disinfect the wire during drilling, pushing wires through the soft tissues to the near cortex, and then tapping through the soft tissues after penetrating the far cortex, predrilling half pins and inserting by hand, use of sharp drill bits, avoiding cannulated drills, low speed pulsatile drilling, irrigating during drilling, avoiding unicortical pin placement, not drilling while using a tourniquet, using half pins with coatings that promote osseointegration, irrigating the pin site after insertion to remove any particles of bone in the surrounding soft tissue immediately covering pin site and compressing with sterile dressings/sponges after insertion to absorb any blood [11].

Patient education is certainly a critical element to decreasing the risk of pin-tract infection. The patient should be educated to look for and identify factors such as pain at the pin-tract site, edema and redness. They can also be educated on the importance of elevating the limb to reduce edema around the pins [11]. Pin-tract site care is extremely important. 
Pema S (2020) An evaluation of the use of a novel microbicidal liquid polymer for the reduction of pin-tract infection in research participants receiving external fixation following deformity correction and traumatic provisional fixation

\section{Conclusion}

It is apparent from this study that the application of Preventogen, a novel microbicidal liquid that forms and occlusive elastomeric film barrier, when applied as part of a pin-tract care protocol on pin sites, significantly reduces the occurrence of pin-tract infections.

Since reimbursement/cost is a limiting factor for both external fixation and internal fixation, there is a significant opportunity to make a positive impact on the growth of the external fixation market through the reduction of the incidence of pin-tract infection associated with external fixation.

This novel microbicidal liquid polymer is the pathway to this opportunity. In a limited number of studies, application of the microbicidal liquid polymer has shown its effectiveness in dramatically reducing the rate of pin-tract infections related to external fixation. This promising opportunity through the use of this product as standard addition to pin-tract care can have a dramatic impact on the increased adoption of external fixation devices.

\section{References}

1. Lethaby A, Temple J, Santy-Tomlinson J (2004) Pin site care for preventing infections associated with external bone fixators and pins. Cochrane Database Syst Rev: CD004551. [Crossref]
2. Iobst CA, Liu RW (2016) A systematic review of incidence of pin track infections associated with external fixation. J Limb Lengthen Reconstr 2: 6-16.

3. Ceroni D, Grumetz C, Desvachez O, Pusateri S, Dunand P, et al. (2016) From Prevention of pin-tract infection to treatment of osteomyelitis during paediatric external fixation. J Child Orthop 10: 605-612. [Crossref]

4. Kazmers NH, Fragomen AT, Rozbruch SR (2016) Prevention of pin site infection in external fixation: a review of the literature. Strategies Trauma Limb Reconstr 11: 7585. [Crossref]

5. Kaplan SL (2014) Recent lessons for the management of bone and joint infections. $J$ Infect 68: S51-S56. [Crossref]

6. Hoyle BD, Costerton JW (1991) Bacterial resistance to antibiotics: the role of biofilms Progr Drug Res 37: 91-105. [Crossref]

7. Pema S (2018) Retrospective Evaluation of Microbicidal Polymer Dressing for Reduction of Infection Following Defromity Correction Surgery. J Drugs Dermatol 17: 1322-1324. [Crossref]

8. Preventogen IFU. Available at: http://www.preventogen.com/. [Accessed August 12, 2019].

9. Berríos-Torres SI, Umscheid CA, Bratzler DW, Leas B, Stone EC, et al. (2017) Centers for Disease Control and Prevention Guideline for the Prevention of Surgical Site Infection, 2017. JAMA Surg 152: 784-791. [Crossref]

10. https://idataresearch.com/product/external-fixation-market-analysis-united-states2017-2023-medcore/

11. Iobst CA (2017) Pin-track infections: Past, present, and future. J Limb Lengthen Reconstr 3: 78-84.

Copyright: (C2020 Pema S. This is an open-access article distributed under the terms of the Creative Commons Attribution License, which permits unrestricted use, distribution, and reproduction in any medium, provided the original author and source are credited. 